\title{
Binary Liquid Mixtures in Porous Solids
}

\author{
S. M. Alnaimi ${ }^{+}$, J. Mitchell, J.H. Strange and J. B. W. Webber \\ School of Physical Sciences, University of Kent, Canterbury, UK, CT2 7NR \\ ${ }^{+}$Permanent Address: Physics Department, University of Qatar, Qatar.
}

\begin{abstract}
The technique of Nuclear Magnetic Resonance (NMR) cryoporometry has been used to study the behaviour of binary liquid mixtures of water and decane in porous sol-gel silicas. The pore volume occupied by each liquid was measured as the ratio of water to decane was altered. It was observed that the water preferentially absorbed onto the silica surface and so was able to displace the decane from the pores. Water displaced the decane first from the smallest pores with a higher surface to volume ratio. The addition of sufficient water to just overfill the silicas was seen to completely displace the decane already occupying the pores after twenty-four hours.
\end{abstract}




\section{List of Figures}

Figure 1 The cryoporometry melting curves of decane and water mixtures in $100 \AA$ Merck silica. The decane melting region is clearly separate from the water melting region. As the fraction of water increases the decane shifts from the pores into the bulk.

Figure 2 The pore volume distributions for decane (top) and water (bottom) generated from the cryoporometry melting curves in Figure 1 . It can be seen that water preferentially displaces decane from the smallest pores.

Figure 3 Pore volume distributions for decane (top) and water (bottom) mix in $40 \AA$ Merck silica. The water initially occupies pores that are too small for the larger decane molecules to enter, and then displaces the decane as the volume of water is increased. 


\section{Introduction}

Various applications of Nuclear Magnetic Resonance (NMR) have been used to study the behaviour of water and oil mixtures in natural rocks ${ }^{1}$. This has been a source of considerable interest to those studying the removal of oil from oil-bearing rocks by flushing the systems with water. Normally the natural rock samples have extremely broad pore size distributions, ranging from microns to millimetres, limiting the utility of NMR cryoporometry ${ }^{1,2}$ for studying the behaviour of the confined liquids. The most frequently used NMR technique has been relaxometry ${ }^{3}$. Although this method can provide estimates of the pore size distributions in the rocks ${ }^{4}$, it is highly sensitive to surface interactions ${ }^{5}$ and can be difficult to interpret accurately ${ }^{6}$. Diffusion studies of flowing systems using NMR Pulsed Field Gradient (PFG) ${ }^{7}$ measurements have shown how water can partially displace oil from rock pores. These studies all used model immiscible liquid mixtures in real rock systems. In this study cryoporometry has been employed to analyse binary mixture immiscible liquids, water and decane, in porous sol-gel silicas provided by Merck. In this way the pore geometry is already known, allowing the interactions between the water, decane and pore surface to be investigated.

NMR cryoporometry ${ }^{1}$ uses the depression in melting point of a crystal in confined geometry compared to the bulk melting point of the frozen liquid. The depression in melting point is related to the size of the confinement, according to the GibbsThomson equation (1), where $\Delta T_{m}$ is the depression in melting point, $x$ is the pore diameter and $k$ is a constant related to the thermodynamic properties of the absorbate ${ }^{8}$. Cryoporometry is a secondary method for determining pore size distributions because the $k$ value first needs to be calibrated for a given absorbate in a set of test measurements. The cryoporometry measurement is made by recording the signal from the resonant nuclear spins $\left({ }^{1} \mathrm{H}\right.$ protons) in molecules in a liquid state. It is assumed that this signal is proportional to the total liquid volume in the sample.

$$
\Delta \mathrm{T}_{\mathrm{m}}=\frac{\mathrm{k}}{\mathrm{x}}
$$

Cryoporometry has previously been used to study a binary mixture by Valiullin and Furo $^{9}$ who studied a miscible mix of nitrobenzene and n-hexane in porous silica 
glasses. A high-resolution spectrometer was used to allow the NMR signal from the two organic liquids with similar melting points to be spectroscopically distinguished. In this work, a low-resolution spectrometer was used. The melting points of the decane and water were so well separated that they were easily distinguishable in the cryoporometry melting curves.

\section{Experimental}

The measurements were made using a scanning-cryoporometer with a temperature resolution of $1 \mathrm{mK}$, designed and built by Webber $^{2}$. The $k$ value of decane was first calibrated using sol-gel silicas with nominal pore diameters of $60 \AA$ and $100 \AA$ provided by Merck, and $200 \AA$ provided by Unilever. The $k$ value was determined to be $824 \mathrm{~K} \AA$. This was confirmed by experiments using cryoporometry measurements and Differential Scanning Calorimetry (DSC) thermoporometry ${ }^{10}$. Water had been previously shown to have a $k$ value of $583 \mathrm{~K} \AA^{2}$.

The samples were made by first slightly over filling the silica with decane. Water was then added incrementally and a cryoporometry measurement made. The addition of known amounts of water, a little at a time, was conducted until a bulk water melting step was observed in the cryoporometry measurement. The cryoporometry data sets were then smoothed and monotonicity was enforced to prevent negative pore volumes being recorded when the data was differentiated. The data was split appropriately into two temperature ramps to allow pore size distributions to be determined from both the water and decane melting curves.

\section{Results}

The first experiments were conducted in $100 \AA$ Merck silica. This silica had previously been characterised using gas adsorption and cryoporometry with cyclohexane as the absorbate ${ }^{2}$. The cryoporometry melting curves for the binary mixtures can be seen in Figure 1. The added water entered the pores and no bulk water signal was detectable until half of the absorbate mix was water. As the water entered the pores, the decane shifted from the pores into the bulk. This can be seen by a shift in the ratio of the pore melting step and the bulk melting step for the decane. The total decane signal remained approximately constant. Once $50 \%$ of the absorbate (by volume) mix was water, a bulk water step was observed. No more water was 
added after that. However, the sample was re-measured after twenty-four hours where no signal could be observed for confined decane. Instead, the water occupied all of the open pore volume, indicating that the water could displace the decane completely over time.

The pore size distributions taken from the cryoporometry results in Figure $\mathbf{1}$ can be seen in Figure 2. Whereas the cryoporometry melting curve only shows that the water displaces the decane, the pore size distributions show where the water goes. The water preferentially displaces the decane from the smallest pores. For a mix containing $10 \%$ water, pores with a nominal diameter of $80 \AA$ were occupied by water. When the quantity of water was increased to $50 \%$ of the mixture, the nominal pore diameter of pores occupied by the water increased to $95 \AA$. At the same time the width of the pore size distribution occupied by the water increased dramatically. By contrast the decane occupied pores of a nominal diameter of $97 \AA$ when $10 \%$ of the mixture was water; this increased to $160 \AA$ when $50 \%$ of the mixture was water. That meant the remaining decane only resided in the largest pores.

Similar displacement behaviour was observed in the $60 \AA$ and $40 \AA$ Merck silicas. The behaviour in the $40 \AA$ silica differed initially. Examples of the pore size distributions obtained are shown in Figure 3. The pore size distribution obtained from the water signal indicated pores that were smaller than those observed in the pore size distribution from the decane. The nominal diameter of pores occupied by the water was $26 \AA$ in both mix ratios, whereas the pores containing decane increased from an average nominal pore diameter of $36 \AA$ to $49 \AA$ as the quantity of water in the mix was increased. The determination of the pore size distribution for the water was complicated because the depressed melting point at the low end of the distribution (associated with pore diameters $<20 \AA$ ) coincided with the bulk melting step of the decane. Such overlapping of liquid melting steps will always place limitations on the binary mixtures that can be observed by cryoporometry without spectroscopic resolution.

\section{Discussions}

NMR cryoporometry provides a unique method of investigating binary liquid mixtures within pores. Usually the NMR liquid signal (which is dependent on the 
number of nuclear spins) is assumed to be proportional to the total volume of liquid in the sample, so a calibrated pore volume can be calculated, assuming the density of the liquid is constant ${ }^{1,2}$. When two liquids are present, the NMR signal will be weighted depending on the spin density of each liquid. A decane molecule contains eleven times the number of spins (protons) as a water molecule. However, the density of the liquid decane is approximately $3 / 4$ of that of water at room temperature. Without knowing the molecular densities of the liquids, nor how they differ in the pores from the bulk, it becomes very difficult to accurately scale the pore volumes. Therefore, the pore volume scales (y-axes) in Figure 2 and Figure 3 have been left as the measured value in arbitrary units and not rescaled as a calibrated volume.

The results of this study clearly show that water can displace decane from porous solgel silicas, given suitable time for the displacement to occur. It has been shown elsewhere that water has a far stronger affinity to the surface in these silicas than nonpolar organic liquids ${ }^{5}$. This is because the silica surface contains hydroxyl groups to which the water molecules can form hydrogen bonds. It was therefore reasonable to expect the water to preferentially enter the smallest pores (with the greatest surface to volume ratio) first, displacing the decane. This may also explain why the water appeared to find pores in the $40 \AA$ that were unseen by the decane: the water will be attracted to the surface and drawn into cracks that the larger non-polar decane molecules could not enter without being forced. The next step in this study would be to explore the behaviour of this binary mixture in a hydrophobic surface modified silica.

We conclude that NMR cryoporometry provides a powerful quantitative method for the investigation of binary fluid mixtures in porous media.

\section{Acknowledgments}

The authors are grateful to EPSRC for supporting this work. 


\section{References}

1 J. H. Strange, M. Rahman, and E. G. Smith, Physical Review Letters 71 (21), 3589 (1993).

2 J. B. W. Webber, PhD Thesis, University of Kent, 2000.

3 R. J. S. Brown and I. Fatt, Petroleum Transactions 207, 262 (1956).

4 M. H. Cohen and K. S. Mendelson, Journal of Applied Physics 53 (2), 1127 (1982).

5 J. H. Strange, J. Mitchell, and J. B. W. Webber, Magnetic Resonance Imaging 21 (3-4), 221 (2003).

6 R. J. S. Brown, G. C. Borgia, P. Fantazzini, and E. Mesini, Magnetic Resonance Imaging 9, 687 (1991).

7 P. J. Barrie, Annual Reports on NMR Spectroscopy 41, 265 (2000).

8 C. L. Jackson and G. B. McKenna, J. Chem. Phys. 93 (12), 9002 (1990).

9 R. Valiullin and I. Furo, Journal of Chemical Physics 116 (3), 1072 (2002).

10 M. Brun, A. Lallemand, J. F. Quinson, and C. Eyraud, Thermochimica Acta 21, 59 (1977). 


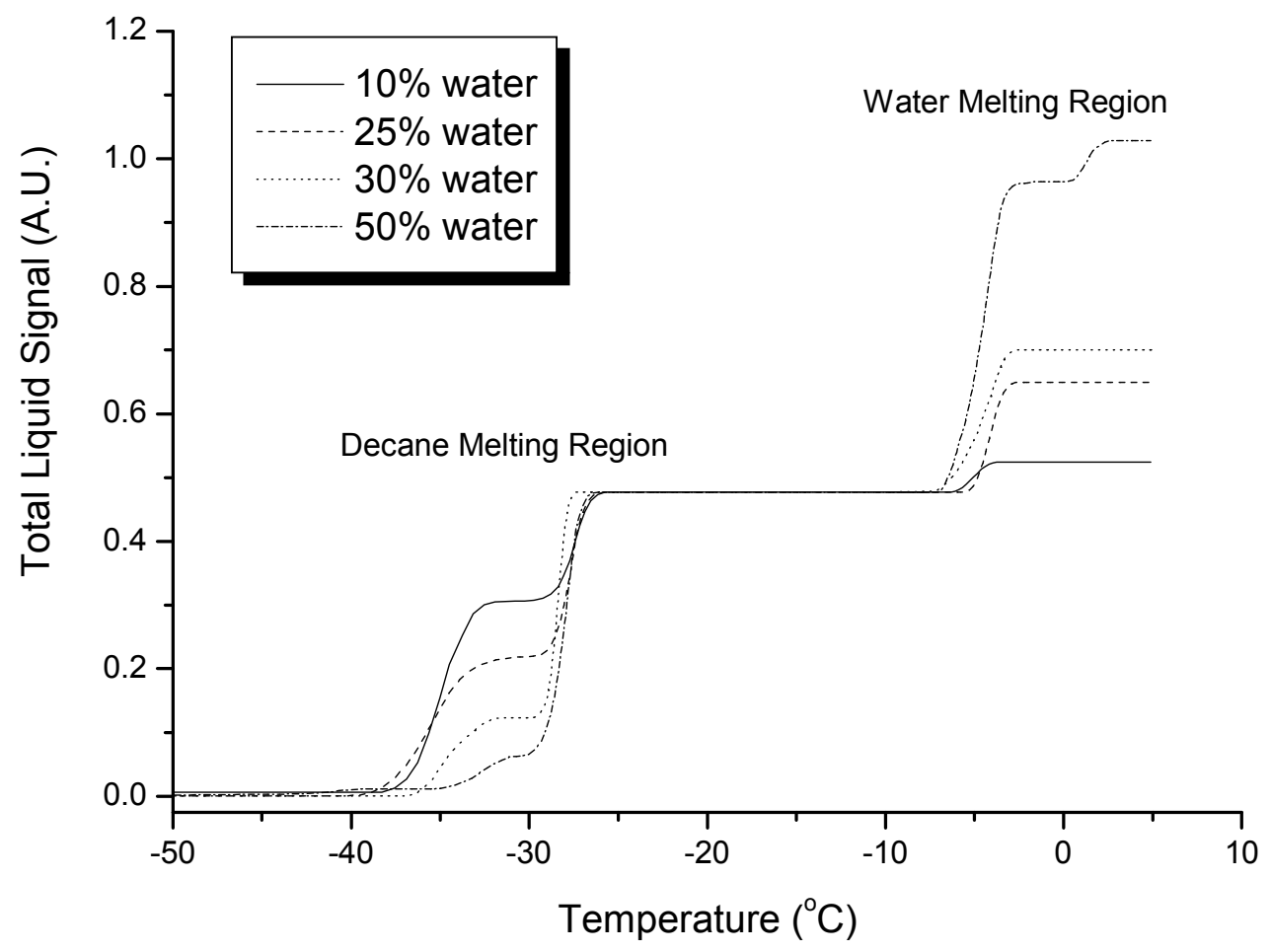

Figure 1 The cryoporometry melting curves of decane and water mixtures in $100 \AA ̊$ Merck silica. The decane melting region is clearly separate from the water melting region. As the fraction of water increases the decane shifts from the pores into the bulk. 


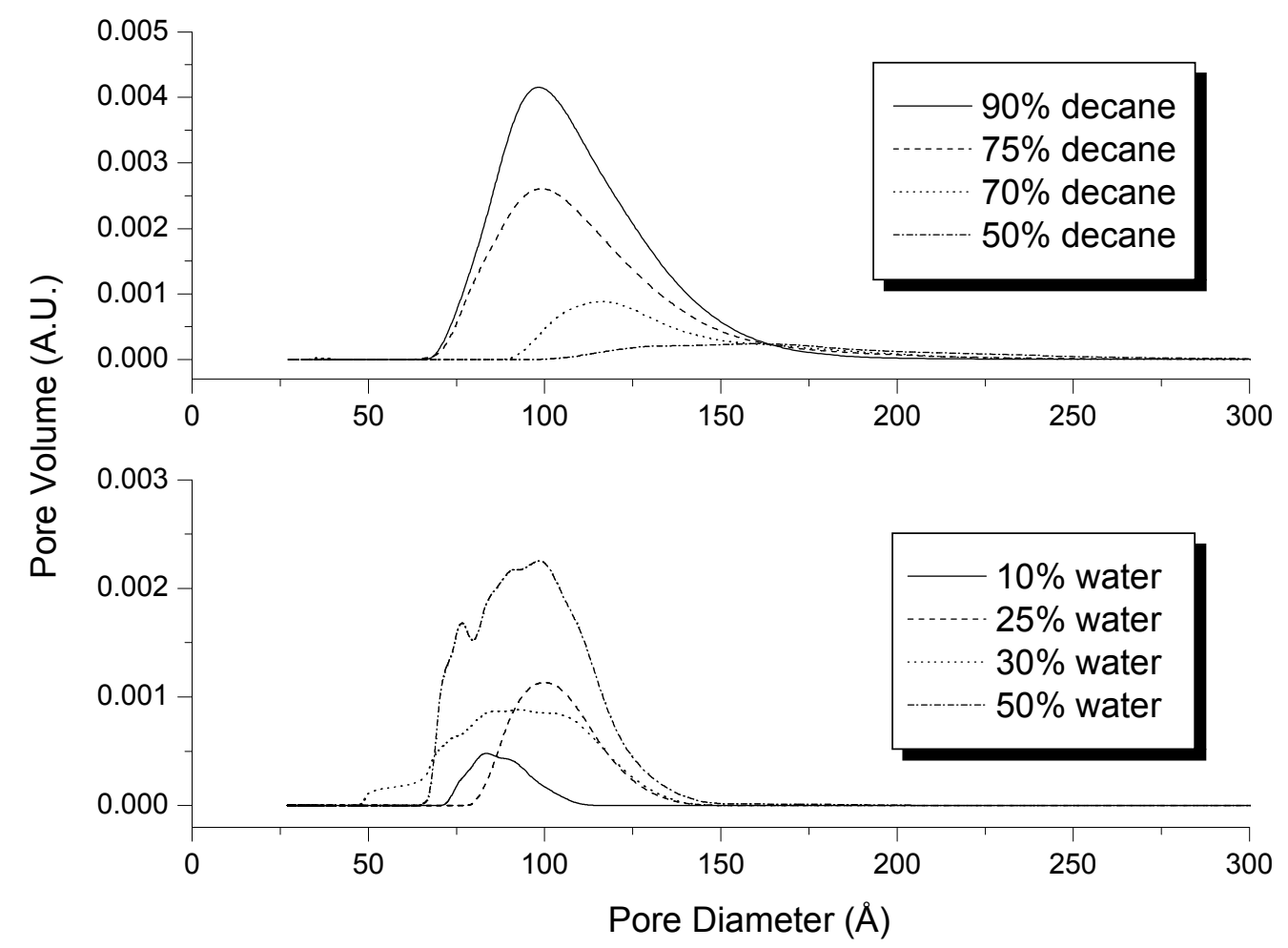

Figure 2 The pore volume distributions for decane (top) and water (bottom) generated from the cryoporometry melting curves in Figure 1. It can be seen that water preferentially displaces decane from the smallest pores. 


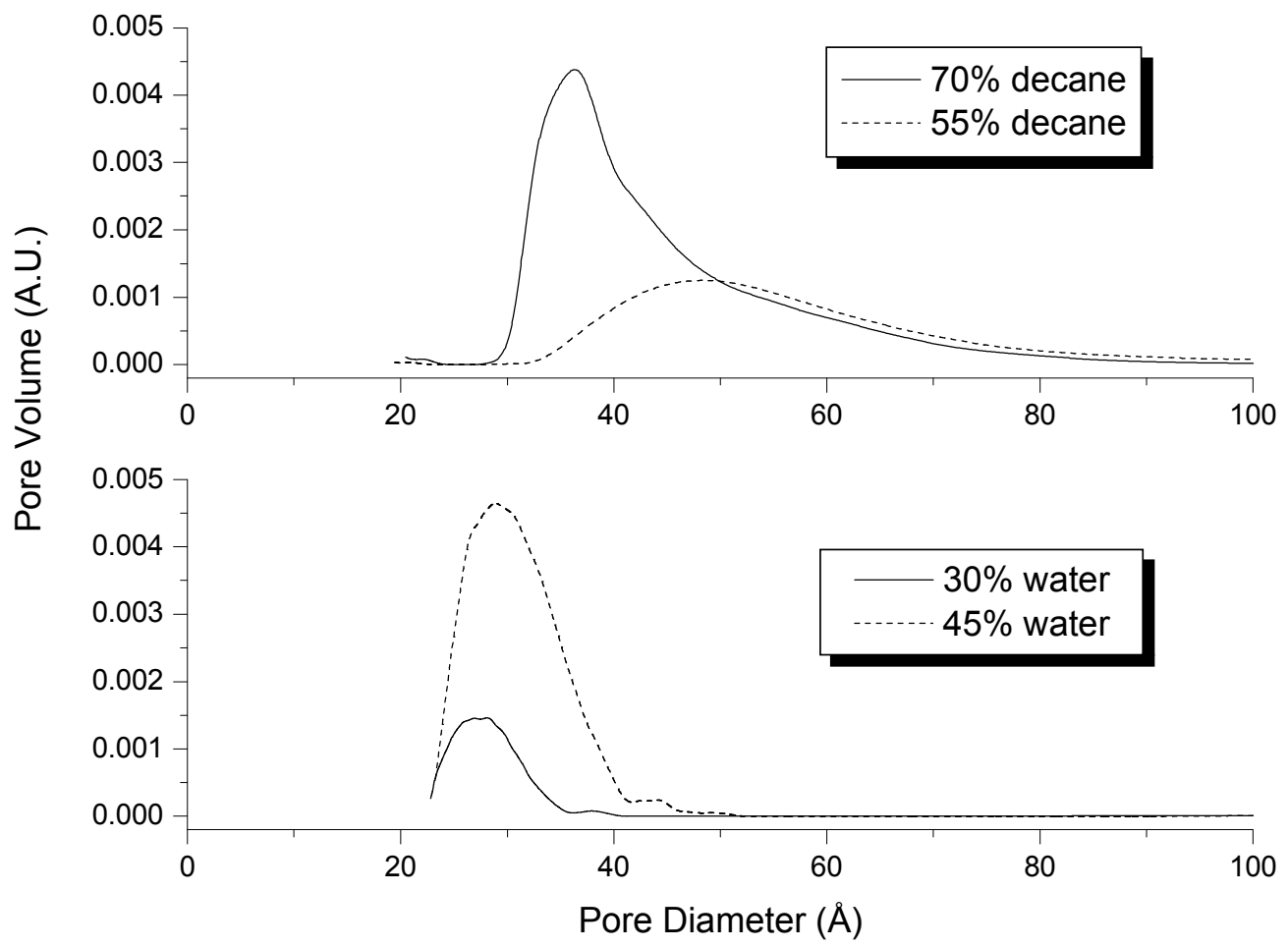

Figure 3 Pore volume distributions for decane (top) and water (bottom) mix in $40 \AA$ Merck silica. The water initially occupies pores that are too small for the larger decane molecules to enter, and then displaces the decane as the volume of water is increased. 